\title{
La edad como factor determinante en la decisión para utilizar la terapia de anticoagulación oral: una auditoria de gestión clínica.
}

\author{
Dr. Nigel P. Murray1, 2, 3,Dra. Esther Meroni L.3 ${ }^{3}$ TM Lic. Ma Mercedes Cárdenas S.4, \\ TM Pamela Cea V.4 \\ ${ }^{1}$ Hospital de Carabineros de Chile, Simón Bolívar 2200 Ñuñoa, Santiago \\ 2Facultad de Medicina, Universidad Mayor, Renato Sánchez Las Condes, Santiago \\ 3 Instituto Nacional de Geriatría, José M. Infante 370 Providencia, Santiago. \\ ${ }^{4}$ Sección Hematología, Hospital de Carabineros de Chile, Simón Bolívar 2200, Nuñoa, Santiago
}

\begin{abstract}
Resumen
Introducción: Con los cambios demográficos, más adultos mayores cumplen con los criterios para la terapia anticoagulante; sin embargo, este grupo etario en particular tiene potencialmente más factores de riesgo para sufrir hemorragias causadas por la terapia anticoagulante. El objetivo de este estudio fue determinar la calidad de control del tratamiento de anticoagulación y su relación con la edad.

Métodos: Se analizaron todos los pacientes atendidos en el Policlínico de Anticoagulación, Hospital de Carabineros de Chile, antes y después de un programa de educación al paciente e implementación de una guía clínica.

Resultados: 188 pacientes, 102 mujeres (edad promedio 71,2 años) y 86 hombres (edad promedio 71,1 años) participaron en el estudio. Todos los pacientes cumplieron con las pautas de la guía. Antes de la implementación, los pacientes > 80 años tuvieron más exámenes con un INR > 5,0; después no hubo diferencias. El análisis post- guía demostró no haber diferencia significativas entre los pacientes según la edad.

Conclusiones: Con la implementación de una guía clínica y educación del paciente, la edad "per se" no es una contraindicación para el tratamiento anticoagulante en pacientes que cumplen con los criterios establecidos.
\end{abstract}

Palabras clave: anticoagulación oral, adulto mayor, control de calidad

\section{Age as a determinant factor in the decision to use oral anticoagulant therapy: clinical auditing of a prospective program for anticoagulation control.}

Background: With the world's population changes more elderly people fulfill the criteria for oral anticoagulation; however, these patients have the highest risk for hemorrhagic side effects.

Aim: to determine the effect of patient age on the quality of oral anticoagulation control.

Patients and Methods: All patients attending the Outpatient Anticoagulation Clinic, Hospital de Carabineros de Chile, were included in the study. Baseline parameters were measured before and after the introduction of a clinical protocol including patient education.

Results: 188 patients, 102 women, mean age 71.2 years and 86 men mean age 71.1 years participated. All patients fulfilled the protocol's criteria. Before the implementation of the program, patients older than 80 years

Correspondencia: Dr. Nigel Peter Murray

Hospital de Carabineros de Chile

Simón Bolívar 2200 Ñuñoa, Santiago

Fono/Fax: 2256168

Correo Electrónico:nigelpetermurray@gmail.com 
were more likely to have occasional INRs $>5.0$ compared to younger patients. After program implementation all age groups significantly improved their control parameters; no differences in time outside the therapeutic range between age groups was observed.

Conclusion: Age "per se" is not a contraindication for oral anticoagulation. With an educational program and a dedicated outpatient clinic, the number of elderly patients achieving therapeutic targets of anticoagulation is the same as that in younger age groups. Thus, older patients who potentially have more to gain from stroke prevention should be considered for anticoagulation when fulfilling protocol criteria.

Key words: Oral anticoagulation, elderly, quality control

Recibido el 19 de marzo de 2009. Aceptado el 8 de julio de 2009

Rev Chil Cardiol 2009; 28: 363-368

\section{Introducción}

El progresivo envejecimiento de la población chilena ha significado que el número de adultos mayores que cumplen con los criterios para el tratamiento de anticoagulación oral (TACO) para tratar o para prevenir trombo embolia, como en la fibrilación auricular, ha aumentado significativamente. Un $70 \%$ de los pacientes con fibrilación auricular son mayores de 65 años, con una edad promedio de 75 años, y un riesgo de accidente vascular encefálico de 5-7\% por año1. El consenso es que todos los pacientes mayores de 75 años deben ser considerados para el $\mathrm{TACO}^{2}$. El adulto mayor tiene una elevada prevalencia de pluri-patología y en conjunto con la polifarmacia dificulta el control de anticoagulación. Los anticoagulantes orales (ACO) necesitan un monitoreo y ajuste de la dosis frecuentemente para mantener su acción terapéutica y para minimizar los eventos hemorrágicos. Este grupo de pacientes es especialmente sensible a los ACO y pequeños incrementos en la dosis o la introducción o suspensión de fármacos, el uso de hierbas y algunos alimentos pueden causar cambios significativos en el efecto anticoagulante.

Los ACO tienen interacciones con una amplia variedad de fármacos, la mayoría de estos causan un aumento en el efecto anticoagulante, especialmente en el caso de analgésicos y antibióticos, comúnmente usados por el adulto mayor.

Es importante notar que a mayor edad, el riesgo del TACO aumenta, hay más riesgo de sangrado debido a factores múltiples: mayor fragilidad vascular, mecanismos hemostáticos menos eficientes y más patología potencialmente sangrante. Tienen una frecuencia mayor de caídas, trastornos de memoria que pueden afectar, tanto la toma correcta de los fármacos como los controles del TACO, reciben múltiples fármacos, con una tasa de depuración menor debido a una reducción de los procesos metabólicos e interacciones medicamentosas. Hay un aumento en el riesgo de hemorragia en pacientes con TACO con el envejecimiento ${ }^{3,4}$, el estudio de la prevención de accidentes vasculares encefálicos en fibrilación auricular ${ }^{5}$ concluyó que el riesgo de una hemorragia en pacientes con TACO aumentó en relación con la edad, especialmente en aquellos mayores de 75 años. Un riesgo aceptable de hemorragia intracraneal es entre $0,4 \%$ a $0,8 \%$, que significa un control perfecto, este riesgo se duplica en pacientes que sufren caídas, en pacientes con una enfermedad neuro-psiquiátrica, un accidente vascular encefálico previo y se triplica en los pacientes mayores de 75 años 6 .

En el Hospital de Carabineros de Chile una guía de anticoagulación oral fue implementada en diciembre de 2007, basada en los criterios de la Sociedad de Hematología del Reino Unido 7 , cumpliendo con los criterios para anticoagulación crónica, en conjunto con una auditoría continua para cumplir con los estándares nacionales e internacionales. Presentamos los resultados de la implementación de la guía en el Policlínico de TACO en el Hospital de Carabineros de Chile, el control de calidad según las pautas establecidas y la relación con la edad. 
La edad como factor determinante en la decisión para utilizar la terapia de anticoagulación oral: una auditoria de gestión clínica.

\section{Métodos y pacientes}

Un estudio prospectivo, descriptivo y transversal realizado en el Hospital de Carabineros de Chile, Santiago, durante 2 períodos; entre enero y marzo de 2008 como período pre-guía y entre agosto y septiembre de 2008 como período post-guía. Se incluyeron todos los pacientes atendidos en el Policlínico de TACO, se recogieron mediante una planilla de Windows Excel los datos relativos al sexo, edad $<60$ años, 61 a 70 años, 71 a 80 años y > 80 años.

Los indicadores seleccionados para el control del TACO de los pacientes atendidos en el Policlínico fueron: cumplimiento con la indicación de TACO según la guía del Hospital de Carabineros:

a) Indices globales: para los meses de febrero y marzo de 2008 como pre-guía, y los meses de agosto y septiembre de 2008 como post-guía; el mes de noviembre de 2007 fue seleccionado al azar como control histórico. El porcentaje de todos los exámenes con un INR igual o menor de 1,5 unidades y más de 5,0 unidades, fue registrado. Un INR $<1,5$ fue considerado como una anticoagulación inadecuada con riesgo de trombosis y un INR $>5,0$ fue considerado como un nivel de anticoagulación asociado a un riesgo de hemorragia elevada.

b) Indicadores centinelas: los pacientes atendidos en marzo de 2008 tuvieron un análisis de los resultados de los controles entre enero y marzo de 2008, y entre junio y agosto de 2008, respectivamente, utilizando el programa InfoLab ${ }^{\circledR}$. Fue calculado el porcentaje de tiempo dentro de los siguientes parámetros, entre $\pm 0,5$ unidades INR de la pauta recomendada, $\pm 1,0$ unidades INR de la pauta, con un INR $>5,0 \mathrm{y}<1,5$.

c) Número de eventos hemorrágicos y severidad, una hemorragia severa será definida cuando se requiera tratamiento con transfusiones, hospitalización, o en el caso de una hemorragia intra-craneal cuando ocasione una disminución en la funcionalidad del paciente y la muerte.

Análisis estadístico: para las variables demográficas se utilizaron estadísticas descriptivas, para la comparación de proporciones, la Prueba de Chi-cuadrado de 2 colas, y para la comparación de promedios, la Prueba de T-Student de 2 colas. Se consideró un error alfa de 0,05 , un error beta de 0,20 y $p<0,05$ como significativos. Todas las comparaciones se hicieron utilizando el grupo de menor edad, $<60$ años, como referencia.

\section{Resultados:}

Durante el período de estudio fueron evaluados 188 pacientes, 102 mujeres con una edad promedio de $71,2 \pm$ DE 12,6 años y 86 varones con una edad promedio de $71,1 \pm 12,3$ años. Para los grupos etarios, hubo 33 pacientes $<60$ años, 42 entre 61 y 70 años, 64 entre 71 y 80 años y 49 pacientes mayores de 80 años.

Todos los pacientes cumplieron con los criterios de la guía, 13 pacientes recibieron TACO por una trombosis aguda y 175 reciben TACO de por vida, de los cuales el $95 \%$ es por fibrilación auricular crónica.

(a) Índices globales: (Tabla I) El número de exámenes fuera de rango fue más elevado en los pacientes mayores, en comparación con los pacientes menores de 60 años, con un INR sobre 5,0 unidades INR y bajo 1,5 unidades INR, pero esta diferencia desapareció cuando se consideró el número de pacientes con el examen $>5,0$ y $<1,5$ (Tabla II). Comparando entre noviembre de 2007 y marzo de 2008, hubo una disminución significativa en el número de exámenes y pacientes con un INR $>5,0(p<0,02)$ y menor que $1,5(p<0,02)$, en todos los grupos etarios.

(b) Indicadores centinelas: (Tabla III). No hubo diferencias entre los distintos grupos etarios para el porcentaje de tiempo entre el rango $\pm 0,5$ unidades de la pauta, $\pm 1,0$ unidad de INR de la pauta 0 porcentaje de tiempo con un INR menor que 1,5. El grupo $>80$ años tuvo un valor del porcentaje de tiempo $>5,0$ significativamente más elevado que el grupo $<60$ años ( $p=0,016,2$ colas T-Student). Después de la implementación de la guía y el programa de educación al paciente, no hubo diferencias entre los grupos. Comparando los grupos pre y post guía por grupo etario, hubo una disminución en el porcentaje del tiempo con un INR mayor que 5,0 en los grupos de $61-70$ años $(p<0,05)$ $y>80$ años $p<0,01$ ), no hubo otras diferencias significativas. 
N. P. Murray, E. Meroni, Ma M. Cárdenas, P. Cea.

Tabla I: Comparación del número de exámenes entre los rangos $>5,0$ y <1,5, según la edad.

\begin{tabular}{lcccc}
\hline & $<60$ años & $\mathbf{6 1 - 7 0}$ años & $\mathbf{7 1 - 8 0}$ años & $>80$ años \\
\hline INR $>\mathbf{5 , 0} *$ & 2 & 3 & 14 & 8 \\
INR $<1,5^{* *}$ & 13 & 26 & 48 & 34 \\
$N^{*}$ exámenes & 33 & 42 & 64 & 49 \\
\hline
\end{tabular}

* $<60$ años vs $71-80$ años $p<0,05$ vs $>80$ años $p<0,05$

61 - 70 años vs 71 - 80 años $p<0,05$, no hubo diferencias significativas entre los otros grupos para el INR $>5,0$

** $<60$ años vs $71-80$ años $p<0,01$ vs $>80$ años $p 0,01$

No hubo diferencias significativas entre los otros grupos para el INR $<1,5$

Tabla II: Comparación de índice global de control según la edad: № de pacientes según el nivel de INR.

\begin{tabular}{lcccc}
\hline & $<\mathbf{6 0}$ años & $\mathbf{6 1 - 7 0}$ años & $\mathbf{7 1 - 8 0}$ años & $>\mathbf{8 0}$ años \\
\hline INR $>\mathbf{5 , 0}$ * & $1(3,0 \%)$ & $4(9,5 \%)$ & $4(6,3 \%)$ & $4(8,5 \%)$ \\
INR $<1,5 * *$ & $11(33,0 \%)$ & $16(38,0 \%)$ & $24(37,5 \%)$ & $22(46,8 \%)$ \\
N $^{*}$ exámenes & 33 & 42 & 64 & 49 \\
\hline
\end{tabular}

* No hay diferencias significativas entre los diferentes grupos por edad (Chi - cuadrado 2 colas)

Tabla III: Indicadores centinelas pre - guía según edad.

\begin{tabular}{|c|c|c|c|c|}
\hline & $<60$ años & 61 - 70 años & 71 - 80 años & $>80$ años \\
\hline$\%$ Tiempo dentro rango $\pm 0,5$ INR & $38,4 \pm \mathrm{DE} 25,6$ & $39,8 \pm \mathrm{DE} 23,2$ & $35,8 \pm$ DE 20,4 & $33,2 \pm \mathrm{DE} 28,3$ \\
\hline$\%$ Tiempo dentro rango $\pm 1,0$ INR & $74,3 \pm \mathrm{DE} 17,8$ & $63,8 \pm$ DE 27,5 & $68,2 \pm \mathrm{DE} 22,2$ & $64,9 \pm \mathrm{DE} 25,1$ \\
\hline$\%$ Tiempo INR > 5,0 & 1,22 & 2,33 & 3,26 & $7,37^{*}$ \\
\hline$\%$ Tiempo INR $<1,5$ & 12,0 & 18,9 & 15,6 & 13,1 \\
\hline $\mathrm{N}^{0}$ pacientes & 33 & 42 & 64 & 49 \\
\hline
\end{tabular}

* Comparación entre el grupo < 60 años vs $>80$ años p=0 016 (Chi - cuadrado. 2 colas)

Hubo 13 pacientes con fibrilación auricular crónica y un deterioro cognitivo, moderado a severo, definido con un Test de Pfieffer $\geq 5^{2}$, estos pacientes recibieron aspirina en dosis de $325 \mathrm{mg} /$ día en reemplazo del TACO.

No hubo casos de hemorragias moderadas o severas que requiriesen hospitalización o transfu- siones; hubo dos casos en los cuales se indicó la administración de vitamina $\mathrm{K}$, en pacientes de 57 y 62 años respectivamente, en las primeras 2 semanas de tratamiento. Siete pacientes tuvieron equimosis sobre lo normal y 1 sufrió epistaxis leve, ninguno de los pacientes tuvo un INR fuera del rango terapéutico al momento del evento. 
La edad como factor determinante en la decisión para utilizar la terapia de anticoagulación oral: una auditoria de gestión clínica.

Tabla IV: Indicadores centinelas post - guía según edad

\begin{tabular}{|c|c|c|c|c|}
\hline & $<60$ años & $61-70$ años & 71 - 80 años & $>80$ años \\
\hline$\%$ Tiempo dentro rango $\pm 0,5$ INR & $45,0 \pm \mathrm{DE} 33,5$ & $43,0 \pm \mathrm{DE} 32,0$ & $44,5 \pm$ DE 28,5 & $33,7 \pm \mathrm{DE} 28,5$ \\
\hline$\%$ Tiempo dentro rango $\pm 1,0$ INR & $80,9 \pm$ DE 25,0 & $74,3 \pm$ DE 29,2 & $69,9 \pm \mathrm{DE} 31,5$ & $66,7 \pm \mathrm{DE} 26,6$ \\
\hline$\%$ Tiempo INR > 5,0 & 0 & 0 & 3,05 & 1,29 \\
\hline$\%$ Tiempo INR $<1,5$ & 9,0 & 14,5 & 14,1 & 19,9 \\
\hline $\mathrm{N}^{\circ}$ pacientes & 33 & 42 & 64 & 49 \\
\hline
\end{tabular}

No hubo diferencias significativas entre los grupos para cada parámetro (Chi - cuadrado 2 colas)

\section{Discusión}

Desde la implementación de la guía clínica en el mes de diciembre de 2007, todos los pacientes enviados para ser tratados con TACO cumplieron con las pautas y las indicaciones para anticoagulación, incluyendo una evaluación basada en la funcionalidad física y cognitiva. Los pacientes con un deterioro cognitivo significativo, definido con un Test de Pfieffer $>5$, fueron excluidos del TACO, excepto en el caso de una trombosis aguda, y recibieron aspirina en dosis de $325 \mathrm{mg} /$ día, según las recomendaciones de Bellelli et al8.

Los resultados muestran que en las personas $\sin$ deterioro cognitivo moderado o severo, se puede mejorar los resultados del control de TACO con un programa de educación que incluyan al apoderado del paciente. El número de pacientes con INR > 5,0 $y<1,5$ unidades de INR disminuyó significativamente $(p<0,02$ y $p<0,02$ respectivamente). EI mejoramiento ocurrió en todos los grupos. No hubo diferencias significativas en el porcentaje de pacientes en los rangos $>5,0$ y $<1,5$ unidades de INR según la edad. Estudios han concluido que la intensidad de anticoagulación es asociada con el riesgo de hemorragia 9,10 , especialmente con un INR $>5,0$. Con educación y un control estricto cada 2 semanas cuando el control es inadecuado, el riesgo de sobre-anticoagulación ha disminuido significativamente $\mathrm{a}<3 \%$ en todos los grupos. La cantidad de control es igual para todos los grupos en términos globales.
El análisis de los indicadores centinelas en el período pre-guía mostró que aquellos pacientes mayores de 80 años estuvieron un tiempo con un INR $>5,0$ lo cual es significativamente mayor que los otros grupos, con la implicancia de un mayor riesgo de hemorragia. El estudio de la prevención de accidente vascular en pacientes con fibrilación auricular 5 concluyó que el riesgo de hemorragia fue mayor en pacientes mayores de 75 años. Después del programa de educación no hubo diferencias.

Estudios internacionales han reportado un porcentaje de sobre-anticoagulación, definido como el porcentaje del tiempo con un INR sobre 5,0 unidades de entre $1-17 \%^{3,11}$, y niveles de anticoagulación subterapéutica, definido como el porcentaje de tiempo con un INR $<1,5$ unidades en el adulto mayor de entre $37 \%-45 \%$ en casas de reposo 12,13 . Nuestras cifras de $<2 \%$ y $<20 \%$ respectivamente para el grupo de adultos mayores de 80 años, significan que el riesgo de hemorragia o trombosis causado por niveles sub-terapéuticos puede ser minimizado. Por lo tanto, la edad "per se" no es una contraindicación para el TACO en los adultos mayores, sin un deterioro cognitivo moderado o severo. Como consecuencia, proponemos que todos los pacientes que cumplen con los criterios deben ser considerados para TACO independiente de su edad, pero es indispensable una evaluación de la funcionalidad física y cognitiva, pacientes con un Test de Pfeiffer $\geq 5$ deberían recibir aspirina en dosis de $325 \mathrm{mg} / \mathrm{día}$. Los otros pacientes con un control adecuado tienen un bajo riesgo de sufrir las complicaciones potenciales de TACO. 


\section{Referencias}

1. WOLF PA, ABBOTT RD, KANNEL WB. Atrial fibrillation: a major contributor to stroke in the elderly: the Framinghamstudy. Arch Int Med 1987; 147: 1561-4.

2. ALBERS GW, DALEN JE, LAUPACIS A, MANNING WJ, PETERSEN P, SINGER DE. Antithrombotic therapy in atrial fibrillation. Chest 2001; 119: 194S-206S.

3. FIHN SD, CALLAHAN CM, MARTIN DC, MCDONELL MB, HENIKOFF JG, WHITE RH. The risk for and severity of complications in the elderly treated with warfarin: the National Consortium of Anticoagulation Clinics. Am J Med 1996; 124: 970-979.

4. LANDEFELD CS, GOLDMAN L. Major bleeding in outpatients treated with warfarin: incidence and prediction of risk at the start of outpatient therapy. Am J Med 1989; 96: 144-152.

5. Stroke Prevention in Atrial Fibrillation Investigators. Risk factors for thromboembolism during aspirin and warfarin therapy in patients with atrial fibrillation. J Stroke Cerebrovasc Dis 1995; 5: 147-157.

6. Stroke Prevention in Atrial Fibrillation Investigators. Bleeding during antithrombotic therapy in patients with atrial fibrillation. Ann Int Med 1996; 156: 409-16.

7. Hematology Task Force, British Society of Haematology.
Guidelines on oral anticoagulation 3rd edition. Brit J Haematol 1998; 101: 374-387.

8. BELLELLI G, BIANCHETTI A, TRABUCCI M. Anticoagulation to prevent stroke in atrial fibrillation: Comprehensive geriatric evaluation should be routine before treatment is started. BMJ 2000; 321: 1157.

9. HULL R, HIRSH J, JAY R, CARTER C, ENGLAND C, GENT $M$, et al Different intensities of oral anticoagulation in the treatment of profilaxis and thrombosis. N Engl J Med. 1982; 307: 1676-1681.

10. TURPIE AG, GUNSTENSEN J, HIRSH J, NELSON H, GENT $M$. Randomised comparison of two intensities of anticoagulation therapy after tissue heart valve replacement. Lancet 1988; 1: 1242-1245.

11. The European Atrial Fibrillation Trial Study Group: Secondary prevention in non-rheumatic atrial fibrillation and TIA or minor stroke. Lancet 1993; 342: 1255-62.

12. GURWITZ JH, MONETTE J, ROCHON PA, ECKLER MA, Avorn. Atrial fibrillation andstroke prevention with long term care setting. Arch Int Med 1997; 157: 978-984.

13. LACKNER TE, BATTIS GN. Use of warfarin for non-valvular atrial fibrillation in nursing home patients. A prospective study. Arch Int Med 1995; 4: 1017-1026. 